\title{
Trial Sets Domain
}

National Cancer Institute

\section{Source}

National Cancer Institute. Trial Sets Domain. NCI Thesaurus. Code C95103.

A subject domain utilized for the submission of information encompassing and representing data, vocabulary or records related to trial sets. 